\title{
Una mirada a la evaluación por competencias desde el planteamiento didáctico, en la educación primaria
}

\author{
A look at competency assessment from the didactic \\ approach, in primary education
}

Nancy Milena Gómez Molano

Universidad Metropolitana de Ciencia y Tecnología. Ciudad de Panamá, Panamá

Recibido: 10/08/2021 Revisado: 20/09/2021 Aceptado: 28/09/2021 Publicado: 31/10/2021

\section{RESUMEN:}

El presente texto contiene una mirada rápida de la inmersión del término competencias a la educación y la evaluación por competencias, dentro del aula escolar a partir de la didáctica, entendi$\mathrm{da}$, como los recursos con que el docente hace uso de su pedagogía, la serie de herramientas tecnológicas y materiales que favorecen el aprendizaje desde la manipulación concreta. De allí que las proyecciones son evaluadas a partir de la evaluación formativa de las competencias, que se adquieren mediante la aplicación didáctica de actividades que van desencadenando en el educando, un carácter de aprendizaje significativo, demostrado en el proceso de resolución de problemas planteados y que son resueltos con la ayuda del docente. Este proceso complejo logra en el educando el ser, saber y saber hacer en un contexto determinado, reto lograr que ese conglomerado de diversidad cultural que son los estudiantes, adquiera una educación de calidad para la vida. Palabras clave: Competencias, evaluación y didáctica.

\section{ABSTRACT:}

This text contains a quick look at the immersion of the term competences to education and evaluation by competences, within the school classroom from didactics, understood, as the resources with which the teacher makes use of his pedagogy, the series of technological tools and materials that favor learning from concrete manipulation.Hence, the projections are evaluated from the formative evaluation of the competences, which are acquired through the didactic application of activities that are triggering in the learner, a character of significant learning, demonstrated in the process of solving problems raised and that are solved with the help of the teacher. This complex process achieves in the learner the being, knowing and knowing how to do in a given context, challenge to achieve that conglomerate of cultural diversity that are the students, acquire a quality education for life. Keywords: Competences, evaluation and didactics. 


\section{INTRODUCCIÓN}

El término Competencias ha sido utilizado en educación en muchas partes del mundo, pero no siempre fue así. Su origen no es precisamente educativo y se remota más en la parte industrial, en el desarrollo de procesos relacionados con el trabajo en la década de la Revolución industrial. Además, según la Real Academia de la Lengua Española, la define como:.

"Disputa o contienda entre dos o más personas sobre algo, oposición o rivalidad entre dos o más personas que aspiran a obtener la misma cosa", dando aquí un carácter demasiado tecnicista y del cual, la mayoría de los docentes no estamos de acuerdo, puesto que la vida es más que un llegar a una meta de primero, es un recorrido cooperativo, donde se necesita del engranaje de las demás personas para subsistir.

Sin embargo, la concepción básica del término competencia, al insertarse en la educación ha cambiado a gran medida su carácter competitivo, puesto que según CliméntBonilla, Juan B (2014) "Las competencias, en su carácter de entidades hipotéticas de difícil definición dentro de una teoría científica, representan constructos complejos biológica, psicológica y socialmente integrados, lo que las abre a tantas posibilidades como a situaciones (realidades) concretas pueda vinculárseles" (p.153). Esto abre a formas de interacción en el ámbito educativo con una noción de complejidad más epistémica y participativa, involucrando el carácter social y rezagando un poco la individualidad.

Desde esta perspectiva, en muchos países del mundo, especial aquellos que pertenecen a la Organización para la
Cooperación y el Desarrollo Económicos (OCDE) regulan el sistema Educativo, con el objetivo de proponerlas desde un enfoque integral. Así en Europa, por ejemplo:

En la Ley General de Educación de 1970, los elementos que conformaban lo que se entiende hoy como currículo eran los objetivos y los contenidos; la Ley Orgánica de Ordenación General del Sistema Educativo, de 1990, incorporó como nuevo elemento el de los criterios de evaluación, incorporación que mantenía la Ley Orgánica de Calidad de la Educación, de 2002, y la Ley Orgánica de Educación, de 2006, integra en su concepto de currículo el conjunto de objetivos, competencias básicas, contenidos, métodos pedagógicos y criterios de evaluación. (Álvarez, Pérez y Suárez ,2008, p.9)

Es decir, las competencias en la evaluación en Europa se incluyen desde el 2002, en el 2006 se vincula al Currículo y desde allí se inicia la ejecución desde los planteamientos de las competencias básicas, específicas y ciudadanas en el pénsum académico, emergiendo allí, las planeaciones curriculares en los planes de estudio de cada área y cambiando la misión y visión de cada Institución, atendiendo al enfoque por competencias. Todo ello para estar a la vanguardia del mundo cambiante, que cada vez, con el acceso masivo a la tecnología, se hace evidente y necesario ser un ser humano competente para la vida. Parecido aloque sucedió en Colombia con la Ley General de Educación de 1994.

Quedando así, interpuesta a la práctica escolar desde los "cuatro pilares de la educación" la noción válida frente a la concep- 
ción de competencia, desde una generalidad integradora, como lo postula: Álvarez, Pérez y Suárez (2008) "Entre las distintas acepciones de competencia aquí presentadas, la que más se aproxima al uso de este término en el contexto educativo, es la de "pericia, aptitud, idoneidad para hacer algo o intervenir en un asunto determinado". (p18) Aspecto que se tiene en cuenta en los Estándares básicos de Competencia de Colombia, donde se afirma que: "Una competencia se define como saber hacer en situaciones concretas que requieran la aplicación creativa, flexible y responsable de conocimientos, habilidades y actitudes. La competencia responde al ámbito del saber qué, saber cómo, saber por qué y saber para qué" (MEN 2006, p. 12) Agregando aquí, una acepción integradora del individuo, al reconocer el saber que: como el contenido o conocimiento, el saber cómo, que se relaciona con su puesta en práctica del conocimiento, el saber por qué, donde el sujeto selecciona lo que le conviene y el saber para qué, hace alusión al objetivo. Así de alguna manera convirtiendo el termino competencia en un engranaje armónico que busca en el estudiantado, evaluar de manera integral el proceso de aprendizaje.

Incluyendo en el ámbito educacional un conglomerado de competencias que en ocasiones tiende a convertirse en moda, en la canasta de promoción del tiempo actual. Por eso, las competencias básicas, las competencias específicas, las competencias emocionales, las competencias ciudadanas, las competencias laborales etc., todo se ha convertido en competencias y con estas ha llegado también otra forma de evaluar, la famosa evaluación formativa, cuya finalidad se enfoca en el proceso de aprendizaje del educando, con la idealización de fortalecer desde el paso a paso, el quiebre o dificultad para realzar un plan de mejoramiento actualizado que permita al aprendiz, salir de la debilidad encontrada de manera eficaz y a tiempo. Encaminada a la entrega de evidencias del trabajo realizado, como una manera de certificar la resolución de las actividades planteadas por el educando en la respectiva practica pedagógica.

Sin embargo, la evaluación por competencias entra a contradecir la evaluación formativa, porque con la primera se busca fortalecer desde el proceso, ir mejorando poco a poco los aspectos endebles de la enseñanza, de acuerdo a los resultados de evaluación interna Por el contrario, la evaluación por competencias o evaluación externa, busca ubicar en una recta numérica el resultado final del proceso, situando en un nivel a cada institución del país, haciendo un paralelo comparativo entre los países del mundo. Como lo afirma Climént (2014): "Desde la perspectiva evaluativa, el enfoque de competencias se centra en la definición de los resultados esperados, independientemente de la forma - procesos, medios, recursos, circunstancias, condiciones" (p.158).

Pero, a Colombia no le ha ido bien en términos de resultados, porque se ha visto superado por países como Ecuador. Ocupando los puestos de más débil Según la Revista Semana (2021) los efectos ICFES 2020, no son alentadores, puesto que en algunos aspectos hubo un declive, "el resultado más elevado es Lectura Crítica con 52,2 sobre un máximo puntaje de 100 el menor inglés con 46,9 luego de Sociales y Ciudadanas con 48 puntos sobre 100 (p.3) Situación alarmante para el sector educativo, teniendo en cuenta aspectos trascendentales como la pandemia generada por el COVID 19, que afecta a todos los ámbitos, lo que demuestra que las condiciones sociales y económicas influyen a gran medida en 
los resultados de la evaluación escolar, porque un estudiante con hambre es difícil que adquiera los conocimientos requeridos, cuando no cuenta con las condiciones mínimas para recibir de forma pertinente la educación.

\section{Desarrollo de competencias según la lista de mayor a menor}

Por otro lado, las pruebas "Programa para la evaluación Internacional de Alumnos" (PISA), el panorama no es el más sobresaliente, puesto que, los resultados dan a conocer gran debilidad en los conocimientos demostrados por los estudiantes en la prueba estandarizada, proyectando un retroceso frente a los datos obtenidos en la evaluación anterior, es decir que, en lugar de ir mejorando, el país presenta retroceso, pero en comparación con el 2006, que fue el primer examen, ha mejorado un poco.

$\mathrm{Si}$ bien el rendimiento de Colombia en lectura en PISA 2018 fue menor que el registrado en 2015 , si se considera un periodo más largo, el desempeño medio mejoró en todas las materias -incluso la lectura - desde que el país participó por primera vez en PISA en 2006. (OECD, 2019, p.4)

Es decir, que la calidad de la educación en Colombia no es la mejor, no es la más adecuada según las normas establecidas a nivel mundial por medio de evaluaciones estandarizadas que permiten ubicar a cada país según sus resultados. Demostrando de esa forma que el nivel de competencias de los colombianos es bastante básico y que queda mucho por fortalecer a nivel educativo. Por lo tanto, se hace necesario fortalecer el proceso de las competencias individuales, que Según Climént (2014) "Por su injerencia en problemas y necesidades de todo orden (político, social, cultural, económico, ambiental, ético), las competencias individuales (estandarizadas y no estandarizadas) son indispensables para el funcionamiento adecuado de cualquier sociedad". (p.155). Es decir que, todos los seres humanos que pertenecemos a una sociedad en cualquier aspecto, debemos estar en la capacidad de responder a las necesidades de forma activa y para ello se requiere contar con unas competencias individuales fortalecidas.

Es de recordar, que las pruebas internas o externas se aplican para todos los estudiantes de cualquier lugar del país, sin discriminar si son egresados de escuelas urbanas o rurales. Aspecto que en el caso de la ruralidad en la mayoría de las escuelas las condiciones de tecnología y acceso a la información, es muy débil. De allí la importancia de la competencia del docente, la capacidad de innovar y vincular aspectos del campo para proyectos pedagógicos que permitan en el estudiantado adquirir los desempeños necesarios para ir a la par con los demás estudiantes de Instituciones del centro del país que cuentan con mejores condiciones básicas. Permitiendo de esta manera, fortalecer lo propuesto por: Bloom (1956) "Recordar, comprender, aplicar, analizar, evaluar y crear" en un paso a paso que permita preparase para la vida en sociedad y para la globalización.

\section{DESARROLLO}

Desde este aspecto, el sistema educativo no es una rueda suelta, es un conglomerado que obedece a todos los otros sistemas, político, económicos, culturales y sociales, a la vez a la globalización, porque en los últimos años, todo lo que sucede en el mundo afecta a todos, casos como el clima y la misma salud, se han convertido en asuntos plurales para las personas que habitamos este inmenso y al 
mismo pequeño lugar del universo, llamado tierra. Por eso, varios proyectos en busca de organizar el sistema como el proyecto Tuning, surgen reformas en las universidades de $\mathrm{Eu}-$ ropa y con el pasar de los años, se fue fortaleciendo esta alianza creando varios proyectos pilotos "Sócrates", "Erasmus" "Comenius" y algo de esto fue tenido en cuenta en lo propuesto por la OCDE en la Agenda 2030 con miras a vincular de alguna manera estrategias significativas para mejorar la educación.

Así, por ejemplo, dentro de algunos Ítems tenidos en cuenta en la Agenda 2030 esta el 5, Igualdad de género, aspecto que se está fortaleciendo vastamente en el sistema educativo, los Manuales de Convivencia han incorporado la libertad de expresión en el estudiantado de forma amplia, dándole la posibilidad de escoger su orientación sexual y su estilo de vida, sin causar dificultad a los demás. Además de la inclusión de proyectos de sexualidad y construcción de ciudadanía en todos los Planteles Educativos en busca de brindar la información pertinente para evitar embarazos a temprana edad, enfermedades ETS o el bullying y ciber bullying en el estudiantado.

5.Igualdad de género. Apoyo a la educación de las niñas y las mujeres, la participación y empoderamiento de las mujeres en la ciencia, los medios de comunicación y la cultura y el combate de la violencia contra las mujeres en todas sus formas. (UNESCO 2017, p.7).

Por otro lado, la propuesta 2030 incluye un aspecto bastante ambicioso, como es erradicar la pobreza, mediante la educación de calidad. Este ítem es muy amplio y tiene consigo muchas variables que hacen sea difícil de cumplir, puesto que, aspectos como la poca inversión social en programas como vivienda, salud y alimentación, son cada vez más escasos y aunque organizaciones internacionales como la ONU y otras, invierten en Colombia, las necesidades son muchas y se queda corto el presupuesto para cumplir con todos los objetivos. Así el postulado:

1. Fin de la pobreza. Erradicar la pobreza con el desarrollo de la productividad y las capacidades humanas por medio de una educación de calidad y el desarrollo de habilidades; ciencia, tecnología e innovación; acceso a las TIC y a los medios de comunicación; la gestión sostenible de los recursos terrestres y marinos; protección y promoción del patrimonio cultural y diversidad de expresiones culturales. (UNESCO 2017, p.7)

Se queda en una meta distante, que con los años se va haciendo más amplia, porque sumado a la poca inversión, llega otro flagelo que estanca los procesos y es la corrupción, con esta última la posibilidad de erradicar la pobreza se queda en el sueño de unos pocos, mientras los pobres siguen escalando hacia abajo los índices de miseria, aunque las estadísticas muestren avances, el pueblo colombiano cada día se ve más pobre y con esta llega la dificultad de recibir una educación de calidad.

Sin embargo y pese al panorama desalentador en cifras y proyecciones que muestra el Sistema Educativo, este no para, se reinventa en la incertidumbre con el fin de llegar a todos los colombianos, es así que, la evaluación en la virtualidad, se ha convertido en un desafío para la educación Rural, porque las condiciones tecnológicas no son adecuadas para afrontar una plataforma mundial virtual que permita estar de forma asincrónica la mayor 
parte del tiempo, como sucede en la educación presencial. Pero el docente innovador no se queda cruzado de brazos, busca, se documenta, crea una ruta que le permita llegar de una u otra manera al estudiantado. Por eso, el uso del portafolio digital, las comunicaciones vía Whatssap, las llamadas de voz y la entrega de trabajos impresos a los padres de familia en periodicidad de tiempos, son algunas estrategias que el docente emplea con el ánimo de llegar de la mejor forma a los estudiantes y brindarles una educación que le permita abarcar todos los aprendizajes que deben adquirir en el año escolar. Por tanto, la práctica educativa se ha reinventado, desde la planeación y seguimiento del docente y la ejecución de actividades en casa, apoyadas por los padres de familia.

Alquézar,M. y Buzeki. M, (2017) Reconocemos que la práctica docente es una actividad compleja en la que las preocupaciones habituales se centran en facilitar el aprendizaje, lo cual supone trabajar constantemente en la planificación de los procesos de enseñanza y de aprendizaje, en el enfoque metodológico, en el diseño de actividades, en instancias tutoriales $y$ en la evaluación de los aprendizajes. (p.67)

Es decir, el docente es el activador y mediador de procesos de aprendizaje, para lo cual debe estar en la capacidad de asumir su rol, con toda la responsabilidad que requiere, buscar capacitación en TIC, si lo cree conveniente, leer sobre nuevas estrategias, guías didácticas, proyectos pedagógicos o demás que necesite para cualificar su labor, llevar a los estudiantes un plan de trabajo articulado, que le permita a los estudiantes aprender y utilizar dichos saberes en prácticas cotidianas para la vida misma. Un maestro competente, como lo afirma Alquézar, y Buzeki (2017) "Se comprende que la identidad del «ser» docente parte del reconocimiento de la reflexión sobre uno mismo como herramienta que posibilita generar cambios en el proceso de enseñanza $\mathrm{y}$, por consiguiente, también en el aprendizaje" (p.80). Aparte de autoevaluarse y realizar la planeación fundamentada y contextualizada, el educando debe tener la capacidad de evaluar constantemente para redefinir nuevas formas de enseñanza, acordes a las expectativas que presenta la sociedad. Como lo afirma: Álvarez, Pérez y Suárez (2008):

Esta forma integrada y contextualizada de entender los contenidos del proceso de enseñanza-aprendizaje supone un avance con respecto a la interpretación, con frecuencia compartimentalizada, que se ha hecho de los contenidos conceptuales, procedimentales y actitudinales y recoge tres características relevantes de las competencias: son multifuncionales y transferibles, tienen un carácter dinámico e ilimitado y a la vez son evaluables. (P.48)

Recae en el docente una gran responsabilidad, de incorporar en el aula, una planeación adecuada que indague no solo por los contenidos de las competencias específicas de cada área, sino también, vinculando las competencias emocionales, ciudadanas y el contexto donde se va a realizar el aprendizaje. Por lo tanto, las competencias de los docentes son cada vez más complejas, el uso actualizado de dispositivos móviles como celulares o computadores y el trabajo en plataformas virtuales como zoom, meet, clasrroom, Google entre otros, son los componentes que debe poner en 
práctica el docente diariamente para llegar a los estudiantes y en caso que no se cuente con acceso a estas posibilidades, crear otras formas de llegar ya sea por documentos físicos u otros.

Existen múltiples metodologías para llegar al estudiantado, como proyectos de aula, unidades didácticas, Resolución de problemas, clase invertida, juego de roles y más. En este caso, se podría incluir el Método Lean Startup, que muy seguramente es relevante para un aprendizaje enfocado a la investigación, como lo afirma: Ávalos, C. y Sevillano, M (2018): La filosofía que plantea el método Lean Startup en sus tres etapas y concebida como metodología de aprendizaje en las aulas, puede verse materializada en los procesos de aprendizaje de la siguiente forma: CREAR (Build), MEDIR (Measure) y APRENDER (Learn). (p.436). Esta propuesta es una entre muchas que los docentes podemos trabajar en el contexto escolar, la diferencia es que está enfocada en la creatividad y la evaluación del proceso, lo que hace mas formativa la educabilidad y la enseñabilidad, porque frecuentemente se está debatiendo sobre el avance y proponiendo formas para mejorar.

Sin embargo, hablar de cambio en docentes, es hablar de capacitación y al mismo tiempo de inversión social, que debe ser gestionada por las Secretarias de Educación y estas en varios lugares del País, no son muy diligentes en estos procesos, en palabras de Ávalos, C. y Sevillano, M (2018): es indispensable también que haya creatividad y deseos de lograr formación inclusiva, innovadora que fortalezca las destrezas, habilidades investigativas, llegando al resultado de un profesional con motivaciones al cambio y a la aplicación de los valores sociales y culturales a mediano y largo plazo. Pero aún hay mucho por hacer desde la práctica docente, el proceso de cambio tarda años y algunos colegas se resisten al cambio, el método tradicional puede resultar más controlador y permite tener el poder en la clase en especial en primaria, por tanto, algunos docentes lo aplican constantemente. Como lo afirma: Campechano (2003):

Muchos de nuestros colegas educadores todavía basan su práctica docente en una serie de acciones que tienen en la experiencia de la repetición su premisa fundamental. Si algo no está memorizado, no está aprendido. Bajo esta lógica todo marcha bien en los exámenes cuando se trata de repetir o reproducir lo "visto en clase". El "coco" de los alumnos comienza con los problemas, con las proposiciones que implican operar con las reglas de la lógica (p.36)

Es difícil entender que la educación ha cambiado, pero es imperdible reconocer que el modelo de sociedad "Sociedad del conocimiento o era de la información" está presente en la actualidad y los docentes no podemos quedar rezagados a aceptar que el mundo se volvió cada día más cerca y que la información está a la mano, ahora la competencia es acompañar al estudiantado para que sepan tomar las decisiones pertinentes, escoger la información adecuada para el aprendizaje, además de fortalecer las competencias socioemocionales que buscan la cordura del ser humano, la tranquilidad y la mejor forma de salir de los conflictos. Se hace necesario identificar también que la educación se hizo multicultural, pluricultural y la comprensión intercultural.

Por eso, es indiscutible dentro del aula escolar propiciar ambientes de aprendizaje que favorezcan la práctica, la manipulación 
de los objetos, para que el estudiante inicie un proceso de conocimiento que pueda aplicar en su cotidianidad y no se quede solamente en el conocimiento enciclopédico.

Forero, I. (2009). Los modelos de aprendizaje actuales desarrollan competencias como el aprender haciendo y la capacidad para innovar. En este orden de ideas, la reflexión por el aprendizaje deja de ser una preocupación exclusiva del ámbito de la educación, para extenderse a otros ámbitos, como las organizaciones sociales, económicas y las instituciones gubernamentales. Así pues, la dimensión educativa desempeña un papel determinante en la consecución de los fines propuestos por las comunidades, las organizaciones y las empresas. (p.44)

Es decir que, la educación como se dijo al inicio de este texto, es un engranaje que atiende a las necesidades en especial políticas y económicas del país y del mundo, siendo así la preparadora del perfil ocupacional que necesitan las industrias, o sea, las condiciones básicas a los trabajadores han mejorado desde la revolución industrial, pero la función de la escuela continua siendo parecida, formar mano de obra para los grandes empresarios y así debe ser, una escuela pensada para las necesidades del mundo, pero viene la pregunta ¿Ha cambiado el propósito de cualificar a las personas para que sean críticas y fomenten empresas o solamente continúan incorporando mano de obra económica para los grandes empresarios con programas educativos, para la población vulnerable, como los ofrecidos por el SENA? Es un interrogante difícil de responder, porque es lo que hay y cambiar eso es un imposible.
Sin embargo, desde el aula escolar, antes de llegar a la secundaria y al nivel profesional es necesario motivar en el estudiantado, la capacidad de innovación e interrogación, la necesidad de pensar y ser críticos frente a la vida, los cambios sociales y la cotidianidad, todo esto se puede lograr, fortaleciendo las practicas escolares, a partir de proyectos investigativos que permitan a los estudiantes obtener el hábito para el seguimiento a procesos, aspecto que hace falta motivar desde el primer ciclo, para que los jóvenes estén en la capacidad de seguir un paso a paso, sin desligarse de un proyecto y así visualizar un proyecto de vida. Evitando caer en el mero tecnicismo, en realizar actividades sueltas sin propósitos de fondo para que el estudiantado las vincule a su vida cotidiana. Como lo plantea:

Delors, (1996.): En efecto, el progreso técnico modifica de manera ineluctable las calificaciones que requieren los nuevos procesos de producción. A las tareas puramente físicas suceden tareas de producción más intelectuales, más cerebrales -como el mando de máquinas, su mantenimiento y supervisión- y tareas de diseño, estudio y organización, a medida que las propias maquinas se vuelven más "inteligentes" y que el trabajo se "desmaterializa" (p.4)

Es decir, no podemos disponernos a formar desde el modelo meramente tecnicista, incluir dentro de los currículos escolares y llevar de manera proyectiva a la práctica actividades escolares significativas, que permitan que el estudiantado adquiera las competencias específicas y acompañarlos en cuanto a la comprensión crítica y definición de las mejores 
decisiones para sus competencias emocionales. Es un proceso que inicia desde el hogar, pero como allí no corresponde a la escuela formar, porque este acompañamiento escolar solo se ofrece en la escuela de padres, proyecto que en la mayoría de las escuelas por x o y motivos queda relegado, es en los primeros grados donde los estudiantes pueden iniciar desde la interacción, el deseo por aprender y el descubrimiento, fortalecer la cultura critica, para hacer de la sociedad venidera una mejor versión de lo que es hoy la humanidad.

\section{CONCLUSIONES}

La escuela siempre será el foco, donde se realiza la proyección del ser humano que exige la sociedad, porque es en el sistema escolar donde se forma la mayor parte del tiempo diario, por tanto, en la actualidad corresponde educar hacia el enfoque por competencias que exige vincular el ser, saber y saber hacer en contexto, formar a una persona con capacidades personales y sociales que aporte al país y al mundo.

\section{REFERENCIAS BIBLIOGRÁFICAS}

Alquézar, M. y Buzeki, M. (2017) Saber y hacer: evaluación de la transferencia de conocimientos didácticos a la práctica docente. Praxis Pedagógica. No.20 enero-junio ISSN 0121-1494. pp: 63-84.

Álvarez, Pérez y Suárez (2008) Hacia un enfoque de la Educación en Competencias. Consejería de Educación y Ciencia Dirección General de Políticas Educativas y Ordenación Académica - Servicio de Evaluación, Calidad y Ordenación Académica. Asturias.

Ávalos, C. y García, M. (2018) El desarrollo de competencias investigativas en la formación de estudiantes de la UNED de Costa Rica mediante la metodología Lean Startup. Servicio de Publicaciones de la Universidad de Murcia. Murcia (España) ISSN edición impresa: 1699-2105. ISSN edición web (http://revistas.um.es/educatio): 1989-466.

Ávalos, C. y Sevillano, M (2018) El desarrollo de competencias investigativas en la formación de estudiantes de la UNED de Costa Rica mediante la metodología Lean Startup. Educatio Siglo XXI, Vol. 36 no 3 - 2018, pp. 417-442 417 http://dx.doi.org/10.6018/j/350071.

Bloom, B.S. (1956). Taxonomía de los objetivos educacionales, Manual I: El dominio cognitivo. Nueva York: David McKay Co Inc.

Campechano, J. (2003). Los fines de la educación del futuro. Sinéctica, Revista Electrónica de Educación, (23), 35-42. [Fecha de Consulta 14 de abril de 2021]. ISSN: 1665-109X. https://www.redalyc.org/articulo.oa?id=99815908006.

Climént-Bonilla, Juan B. (2014). Origen, desarrollo y declive de las competencias individuales en tiempos de incertidumbre. Educación y Educadores, 17 (1), 149-168. [Fecha de Consulta 9 de abril de 2021]. ISSN: 0123-1294. https://www.redalyc.org/articulo.oa?id=83430693008. 
Delors, J. (1996): "Los cuatro pilares de la educación" en La educación encierra un tesoro. Informe a la UNESCO de la Comisión internacional sobre la educación para el siglo XXI, Madrid, España: Santillana/UNESCO. pp. 91-103.

Forero, I. (2009). LA SOCIEDAD DEL CONOCIMIENTO. Revista Cientifica General José María Córdova, 5 (7), 40-44. [Fecha de Consulta 14 de Abril de 2021]. ISSN: 1900- 6586. Disponible en: https://www.redalyc.org/articulo.oa?id=476248849007

La Taxonomía de bloom y sus actualizaciones

http://www.eduteka.org/TaxonomiaBloomCuadro.php3

Londoño Orozco, Guillermo and Cano García, Elena, "Formación y evaluación por competencias en educación superior" (2015). Libros en acceso abierto. 50. https://ciencia.lasalle.edu.co/libros/50.

Ministerio de Educación Nacional (2006) Estándares Básicos de Competencias en Lenguaje, Matemáticas, Ciencias y Ciudadanas. Proyecto editorial y coordinación Escribe y Edita. Bogotá Colombia.

Resultados Icfes 2020: ¿cómo les fue a los jóvenes y en qué se rajaron? (25/2/2021) Revista Semana. $\mathrm{N}^{\circ}$ p 1-5. https://www.semana.com/educacion/articulo/resultados-icfes-2020-como-les-fue-a-los-jovenes-y-en-que-se-rajaron/202134/

UNESCO (2017) La UNESCO Avanza La Agenda 2030 para el Desarrollo Sostenible. Organización de las Naciones Unidas para la Educación, la Ciencia y la Cultura. 7, place de Fontenoy, 75352 París 07 SP, Franciahttp://en.unesco.org/sdgs.

OECD (2019), PISA 2018 Results (Volume I): What Students Know and Can Do, PISA, OECD Publishing, París, https://doi.org/10.178. 